International Journal of

Molecular Sciences

ISSN 1422-0067

www.mdpi.com/journal/ijms

Article

\title{
Dielectric Properties of Binary Solvent Mixtures of Dimethyl Sulfoxide with Water
}

\author{
Li-Jun Yang ${ }^{1}$, Xiao-Qing Yang ${ }^{1}$, Ka-Ma Huang ${ }^{1, *}$, Guo-Zhu Jia ${ }^{1}$ and Hui Shang ${ }^{2}$ \\ ${ }^{1}$ College of Electronics and Information Engineering, Sichuan University, Chengdu, 610064, P.R. \\ China \\ ${ }^{2}$ State Key Laboratory of Heavy Oil Processing, China University of Petroleum (Beijing), Changping \\ District, Beijing 102249, P.R. China
}

E-Mails: ljyang320@163.com (L.-J. Y.); xqyang@suc.edu.cn (X.-Q. Y.); yyxxqq_mail@163.com (K.-M. H.); jia1689500@126.com (G.-Z.J.)

* Author to whom correspondence should be addressed; Tel. +86-28-85408779;

Fax: +86-28-85408779

Received: 6 February 2009; in revised form: 8 March 2009 / Accepted: 11 March 2009 / Published: 17 March 2009

\begin{abstract}
In this paper, the dielectric properties of water-dimethylsulfoxide (DMSO) mixtures with different mole ratios have been investigated in the range of $1 \mathrm{GHz}$ to 40 $\mathrm{GHz}$ at $298 \mathrm{~K}$ by using a molecular dynamics (MD) simulation. Only one dielectric loss peak was observed in the frequency range and the relaxation in these mixtures can be described by a single relaxation time of the Davidson-Cole. It was observed that within experimental error the dielectric relaxation can be described by the Debye-like model $(\beta \approx$ 1, S.M. Puranik, et al. J. Chem. Soc. Faraday Trans. 1992, 88, 433 - 435). In general, the results are very consistent with the experimental measurements.
\end{abstract}

Keywords: Binary Solvent; Dielectric properties; Activation energy; MD. 


\section{Introduction}

Over the past few years, the use of microwave heating for promoting organic chemical transformations has been widely accepted by scientists [1,2]. The heating characteristics of a particular material via microwave irradiation are dependent on its dielectric properties. Therefore, the study of materials' dielectric properties seems a crucial need [3].

Dimethylsulfoxide (DMSO) and its mixtures with other solvents (particularly water) have aroused much interest among scientists in the last decades [4-12]. The dielectric behavior of supercooled aqueous solutions of DMSO was investigated by Murthy. It was found that a 2:1 complex that was present (in DMSO solutions) in the liquid state was thermolabile and existed in an undissociated state [13]. The melting temperatures were measured by using the dielectric technique in combination with a differential scanning calorimeter. The equilibrium phase diagram of DMSO was found to be eutectic, with two compounds formed by water and DMSO at the ratios of 3:1 or 2:1 [14]. Rasmussen et al. studied the phase equilibrium and non-equilibrium behaviour of solutions dissolved in water and indicated that the water-DMSO system can, in all proportions, be crystallized completely, and a stable hydrate $\left(\mathrm{DMSO} \cdot 3 \mathrm{H}_{2} \mathrm{O}\right)$ was formed under certain conditions [15]. The energetic and structural properties of DMSO-water clusters in gas-phase by DFT and the polarizable force field have been developed by Zhang et al. The hydrogen bond lengths, binding energies and many-body energies of a series of DMSO-water clusters were calculated using high level ab initio methods [16,17].

In fact, the long-standing interest in all mixed solvents is largely due to their importance as tunable reaction media. The dielectric constant and the relaxation time, as well as the refractive indexes and other transport properties of mixed solvents, can be conveniently tuned by changing the composition [18-20]. From a molecular perspective, solvent mixtures are also interesting because they often exhibit complex structural and dynamical features, especially when the components are capable of specific interactions with one another, which may result in strong interspecies molecular associations.

A dielectric study of DMSO-water using molecular dynamics simulation (MD) is introduced in this paper. The static dielectric constant $\varepsilon_{0}$, dielectric constant at high frequency $\varepsilon_{\infty}$, the relaxation time $\tau$, the Cole-Cole curve and the complex permittivity spectrum have been obtained.

\section{Interaction Potentials and Simulation Details}

The mixtures with DMSO mole fractions $x_{D M S O}\left(x_{D}\right)=0.1,0.21,0.35,0.48$ and 0.91 , the intermolecular potentials are described by site-site interactions given by a sum of Lennard-Jones plus Coulomb terms:

$$
u_{i j}(r)=4 \varepsilon_{i j}\left[\left(\sigma_{i j} / r\right)^{12}-\left(\sigma_{i j} / r\right)^{6}\right]+q_{i} q_{j} / 4 \pi \varepsilon_{0} r
$$

where $r$ is the distance between sites $i$ and $j$ in different molecules, $q_{i}$ and $q_{j}$ are the effective charges of sites $\mathrm{i}$ and $\mathrm{j}, \varepsilon_{i j}$ and $\sigma_{\mathrm{ij}}$ are the energy and distance parameters in the Lennard-Jones potential. They are determined from the Lennard-Jones parameters of the different sites according to the "mixing", rule: 


$$
\sigma_{i j}=\left(\sigma_{i}+\sigma_{j}\right) / 2, \varepsilon_{i j}=\sqrt{\varepsilon_{i} \varepsilon_{j}}
$$

where $q_{i}, \sigma_{i}$ and $\varepsilon_{i}$ for the oxygen, sulfur, and methyl sites respectively in DMSO molecule and oxygen, hydrogen sites in water are shown in Table 1 [21].

Table 1. L-J parameters and partial charges for water and DMSO.

\begin{tabular}{cccc}
\hline & $\varepsilon /\left(\mathrm{kJmol}^{-1}\right)$ & $\sigma /(\mathrm{nm})$ & $q /($ a.u. $)$ \\
\hline $\mathrm{O}$ (water) & 0.65 & 0.3165 & -0.82 \\
$\mathrm{H}$ (water) & 0.0 & 0.0 & 0.41 \\
\hline $\mathrm{O}$ (DMSO) & 0.30 & 0.28 & -0.459 \\
$\mathrm{~S}$ & 1.00 & 0.34 & 0.139 \\
$\mathrm{CH}_{3}$ & 1.23 & 0.38 & 0.16 \\
\hline
\end{tabular}

Simulations reported in this paper were run in the NVT ensemble on systems consisting of a thousand molecules placed in a cubic box with periodic boundary conditions at an average temperature of $298 \mathrm{~K}$. The box length was chosen to match the experimental density of DMSO-water mixtures solvent at $298 \mathrm{~K}$. The Lennard-Jones forces were cut off at half of the box length and long-range Coulomb interactions were treated using the Ewald sum method [22]. The equations of motion were solved using combined SHAKE [23] and leap-frog [24] algorithms with a time step of 1 fs, during uninterrupted production runs lasted about $50 \mathrm{ps}$ for each mixture. Then simulation were run in the NVE ensemble, all the simulations were extended up to $1.2 \mathrm{~ns}$, where the first 50 ps were considered as equilibration. The starting solvent configurations for the five compositions of the mixtures have been chosen according to the specifications given in Table 2 .

Table 2. Compositions of the DMSO-water mixtures. Solvent 1 is DMSO; solvent 2 is water; $x_{i}$ is mole fraction of solvent, $n_{i}$ is number of molecules of solvent $i$ in the cubic simulation cell of edge length $L ; \rho$ is the density at $298 \mathrm{~K}, n_{D}$ is the refractive index.

\begin{tabular}{cccccccc}
\hline Composition & $x_{1}$ & $x_{2}$ & $n_{1}$ & $n_{2}$ & $L(\AA)$ & $\begin{array}{c}\rho^{\mathrm{a}} \\
\left(\mathbf{g} / \mathbf{c m}^{3}\right)\end{array}$ & $\begin{array}{c}\text { Refractive index } \\
\left(n_{D}\right)\end{array}$ \\
\hline 1 & 0.1 & 0.9 & 100 & 900 & 34.05 & $1.0105^{\mathrm{b}}$ & $1.3458^{\mathrm{b}}$ \\
2 & 0.21 & 0.79 & 210 & 790 & 36.76 & $1.0242^{\mathrm{b}}$ & $1.3600^{\mathrm{b}}$ \\
3 & 0.35 & 0.65 & 350 & 650 & 39.0 & 1.0927 & 1.4385 \\
4 & 0.48 & 0.52 & 480 & 520 & 41.38 & 1.0983 & 1.4525 \\
5 & 0.91 & 0.09 & 910 & 90 & 47.93 & 1.0965 & 1.4741 \\
\hline
\end{tabular}

${ }^{\mathrm{a}}$ Reference [25].

${ }^{\mathrm{b}}$ Reference [26]. 


\section{Results and Discussion}

\subsection{The static dielectric constant}

To calculate the effective dielectric constant of a complex solute from computer simulation, it is necessary to map the properties of the solute observed in the simulation onto a simpler geometry, one amenable to analytical treatment. Specifically, the solute is approximated as a spherical cavity of volume $V$ and dielectric constant $\varepsilon$ embedded in a uniform dielectric continuum with dielectric constant $\varepsilon_{R F}$. The charge distribution of the solute is represented as a point charge and point dipole placed at the center of the spherical cavity. From the fluctuations of the solute dipole moment, M, observed during a computer simulation, the absolute temperature, $T$, the volume, $V$, of the solute cavity, and the external dielectric, $\varepsilon_{R F}$, it is then possible to determine the dielectric constant $\varepsilon$ inside the solute cavity:

$$
\varepsilon=\frac{1+\left[\left(<M^{2}>-<M>^{2}\right) /\left(3 \varepsilon_{0} V k_{B} T\right)\right] \bullet 2 \varepsilon_{R F} /\left(2 \varepsilon_{R F}+1\right)}{1-\left[\left(<M^{2}>-<M>^{2}\right) /\left(3 \varepsilon_{0} V k_{B} T\right)\right] /\left(2 \varepsilon_{R F}+1\right)}
$$

where $\mathbf{M}$ is the dipole moment of the computational box, $<>$ denotes Boltzmann ensemble averaging, $\varepsilon_{0}$ is the dielectric constant of vacuum, $k_{B}$ is Boltzmann's constant, and all of the above parameters are used SI units.

Figure 1. MD (full symbols) and Reference [2] and [27] (open symbols) data for the mixture's dielectric constant vs. DMSO mole fraction.

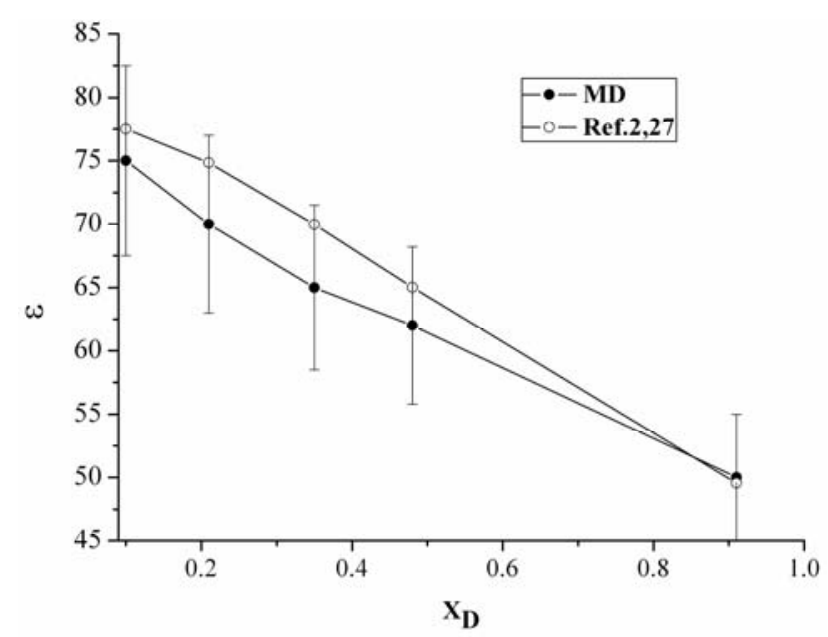

Figure 1 describes the dielectric constant $\varepsilon$ as a function of the composition. The error bars were estimated using the blocking method, considering that the intermolecular potentials used here lack of any polarizability effects beyond the usual dipole moment enhancement and the fact that the SPC/E underestimates water's dielectric constant by about $10 \%$, the MD values of $\varepsilon$ for the mixtures are small than those from Ref. [2] and [27]. 


\subsection{The relaxation time}

Thermodynamic and statistical perturbation theory originally developed by Zwanzig [28] can be used to calculate relative and absolute free energies for molecularly detailed systems [29]. The change of the Gibbs free energy of a system with $\mathrm{N}$ particles at a given temperature, $\mathrm{T}$, and pressure, $\mathrm{P}$, is given by [30]:

$$
\Delta G=-k T \ln <e^{-\Delta V / k T}>_{0}
$$

Where $\Delta V$ is a perturbation of the Hamiltonian.

The composition dependence of the calculated values of $\Delta G$ is given in Figure 2. From this Figure, it can be seen that the free energy of activation increases with the concentration of the nonelectrolyte in water, reaching a maximum value of 3.5 at the concentration fraction of 0.35 , whereafter it decreases. This difference can be attributed to the fact that the components are capable of specific interactions with one another.

Figure 2. Free energy change of activation via water-DMSO solutions vs. mole fractions.

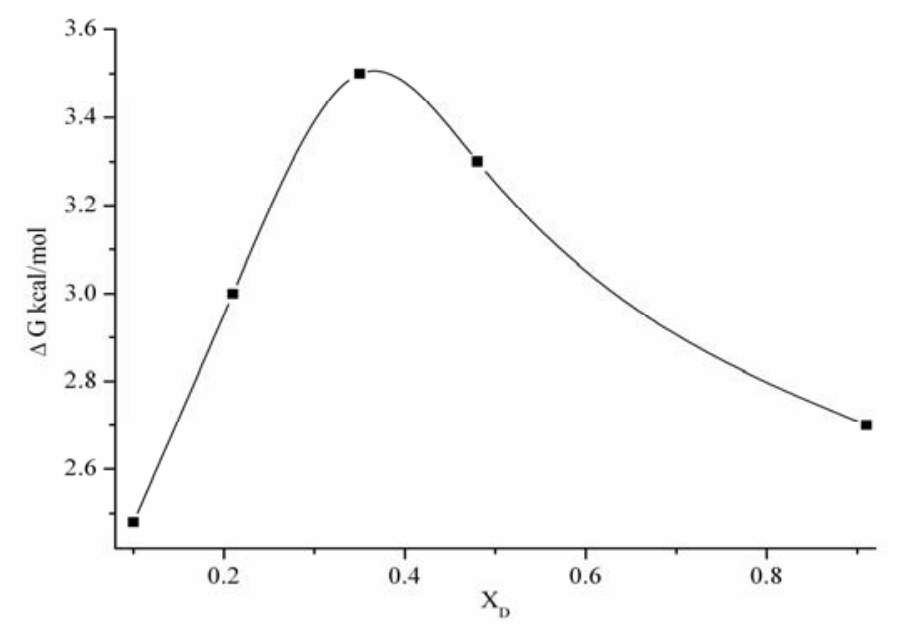

Eyring et al. have applied the theory of rate processes to molecular dipole relaxation with the following equation [31]:

$$
\tau=\left(h / k_{B} T\right) \exp (\Delta G / R T)
$$

where $\Delta G$ is the free energy change of activation for the dipole relaxation, $k_{B}$ is Boltzmann's constant, $h, R$ are Planck's constant and the molar gas constant, respectively.

The mixture's overall dielectric relaxation time $\tau$ is in good agreement with the experimental, except for compositions between 0.3 and 0.42 , where the simulations predict significantly higher relaxation times seen from Figure 3. 
Figure 3. Mole fraction dependence of the relaxation time $\tau$ of DMSO-water mixtures at $298 \mathrm{~K}$.

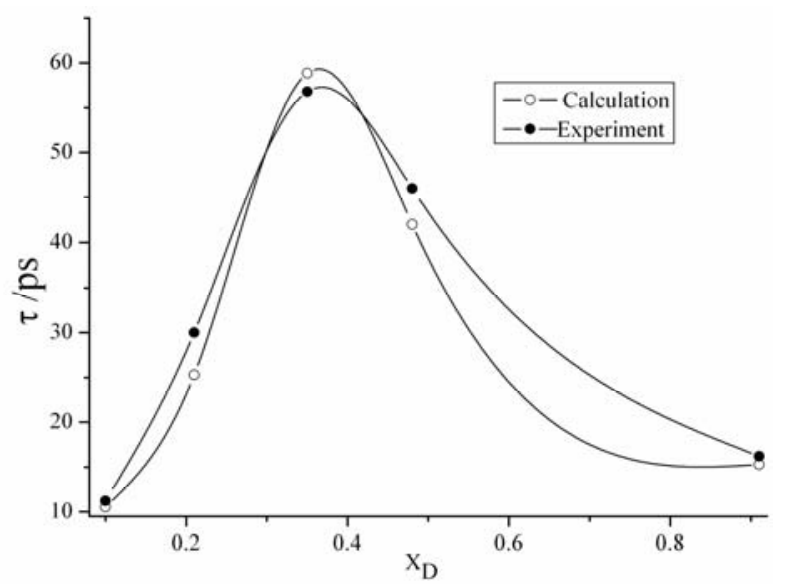

In Figure 3, the open symbols show mole fraction dependence of the relaxation time is calculated by Equation 5 .

\subsection{The permittivities vs. Frequency}

The complex permittivities are fitted by the nonlinear least-squares fit method to the HavriliakNegami expression to obtain various dielectric parameters:

$$
\varepsilon^{*}(\omega)=\varepsilon_{\infty}+\left(\varepsilon_{0}+\varepsilon_{\infty}\right) /\left[1+(j \omega \tau)^{1-\alpha}\right]^{\beta}
$$

Where $\varepsilon^{*}(\omega)$ is the complex permittivity at an angular frequency $\omega, \varepsilon_{\infty}$ is the permittivity at high frequency, $\varepsilon_{\infty}$ is the static dielectric constant, $\alpha$ is the shape parameter representing symmetrical distribution of relaxation time and $\beta$. is the shape parameter of an asymmetric relaxation curve. Equation 4 includes Cole-Cole $(\beta=1,0<\alpha<1)$ [32], Davidson-Cole $(\alpha=0,0<\beta<1)$ [33] and Debye ( $\alpha=0, \beta=1$ ) [34] relaxation models. In our system, it is observed that under experimental error the dielectric relaxation can be described by the Debye-like $(\beta \approx 1[35])$. Therefore, Equation 6 can be rewritten to Equation 7:

$$
\varepsilon^{*}(\omega)=\varepsilon_{\infty}+\left(\varepsilon_{0}+\varepsilon_{\infty}\right) /(1+j \omega \tau)
$$

where the complex permittivity $\varepsilon^{*}(\omega)$ is

$$
\varepsilon^{*}(\omega)=\varepsilon^{\prime}(\omega)-i \varepsilon^{\prime \prime}(\omega)
$$

For the Debye Equation, the real $\varepsilon^{\prime}$ and imaginary $\varepsilon^{\prime \prime}$ contributions to the dielectric behavior can be expressed by:

$$
\begin{aligned}
& \varepsilon^{\prime}(\omega)=\varepsilon_{\infty}+\left(\varepsilon-\varepsilon_{\infty}\right) /\left(1+\omega^{2} \tau^{2}\right) \\
& \varepsilon^{\prime \prime}(\omega)=\left(\varepsilon-\varepsilon_{\infty}\right) \omega \tau /\left(1+\omega^{2} \tau^{2}\right) \\
& \varepsilon_{\infty} \approx 1.1 n_{D}^{2}
\end{aligned}
$$

Permittivity and losses for DMSO-water mixtures at $298 \mathrm{~K}$ are shown in Figure 4, where a single relaxation peak is observed for the entire concentration range of all the DMSO-water mixtures in the 
range of 1 40 GHz. Figure 4(a) obviously indicates that the higher the frequency the lower of the real $\varepsilon$ '. Nevertheless, the real $\varepsilon^{\prime}$ decreases with the concentration of DMSO increasing, however, a completely opposite result were obtained when $x_{D}$ is more than 0.35 . It can be addressed that the real $\varepsilon$ ' of the mixtures strongly depend on the DMSO concentration. It is expected that this phenomenon is due to the cooperative motion of DMSO-water molecules through hydrogen bonds.

Figure 4. Permittivities and losses for DMSO-water mixtures at various concentrations at 298 K. (a) The real vs. frequency. (b) The imaginary vs. frequency.

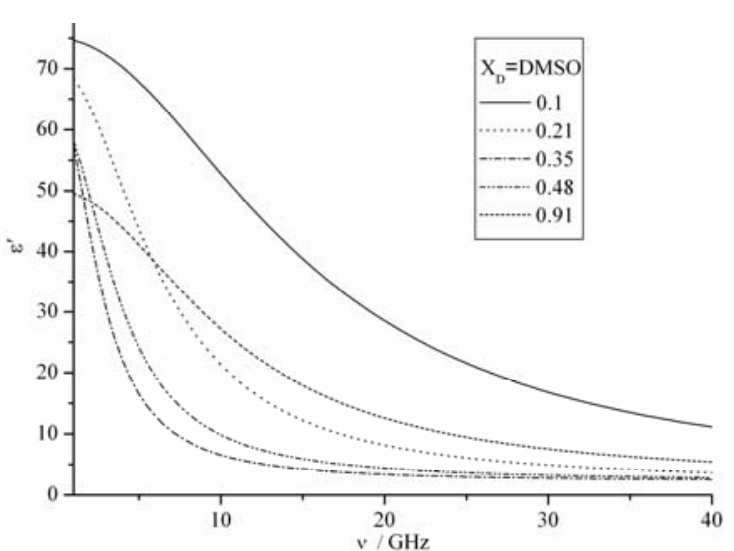

(a)

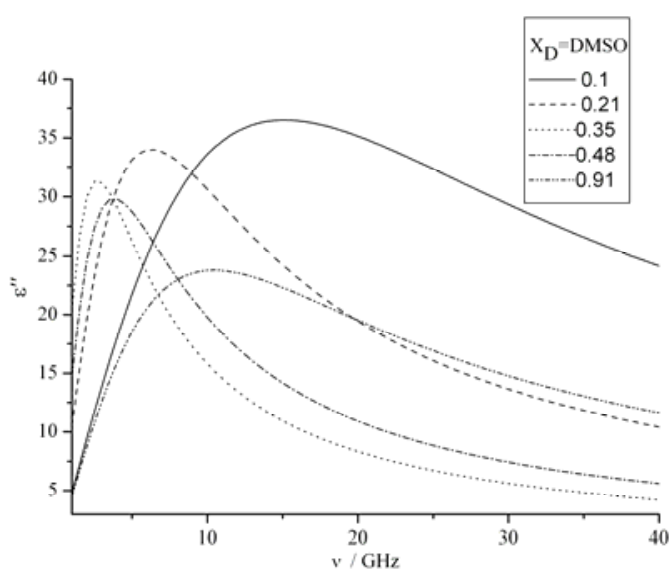

(b)

From Figure 4(b), the imaginary $\varepsilon^{\prime \prime}$ of dielectric constant increases with the frequency till it reaches the summit, after which the imaginary part reduce gradually with the frequency. Generally, the primary process observed for various associated liquids and those water mixtures exhibit an asymmetric loss peak. In a theoretical study, it has been suggested that the motional units in the correlated domains cooperatively move and the heterogeneity of the size distribution of the domain results in the asymmetric shape of the loss peak. The concentration dependence of DMSO-water mixture solution had been calculated by Equation 9, the Cloe-Cloe diagrams can be drawn as shown in Figure 5. It can be concluded that the relation between $\varepsilon^{\prime}$ and $\varepsilon^{\prime \prime}$ are circle, but those centre aren't the cross axle $\varepsilon^{\prime}$.

Figure 5. The Cole-Cole curve of DMSO-water mixture at various concentrations.

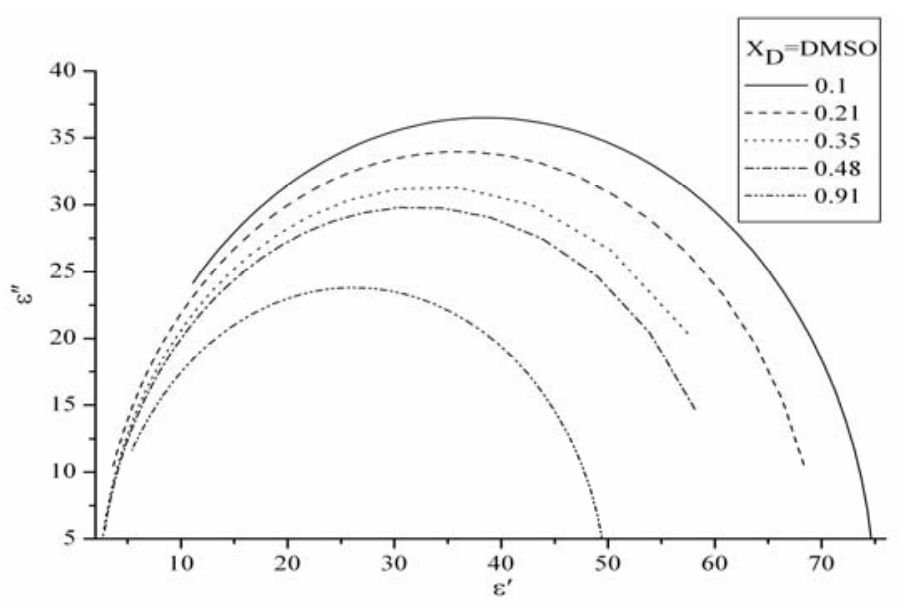




\section{Conclusions}

This paper presents the picosecond dynamical profile of water-DMSO solutions over the entire range of composition. The static dielectric constant, dielectric relaxation in the frequency of microwave, as well as the microwave spectra of DMSO-water mixtures have been investigated through molecular dynamics simulation at room conditions, and this simulation has been proved to be an efficient tool for the study of molecular processes in solutions under the sufficiently wide frequency range.

\section{Acknowledgements}

This project is supported by the National Science Foundation of China under Grant NO.60531010 and Research Fund for the Doctoral Program of Higher Education of China under Grant NO. 20070610120.

\section{References and Notes}

1. Leadbeater, N. Making microwaves. Chem. World 2004, 1, 38-41.

2. Adam, D. Microwave chemistry: Out of the kitchen. Nature 2003,421, 571-572.

3. Jin, Q.H.; Dai, S.S.; Huang, K.M. Microwave Chemistry; Science Press: Beijing, China, 1999.

4. Vechi, S.M.; Skaf, M.S. Molecular-dynamics simulations of dimethylsulfoxide- methanol mixtures. J. Chem. Phys. 2005, 123, 154507-154514.

5. Cowie, J.M.G.; Toporowski, P.M. Association in binary liquid system dimethyl sulphoxidewater, Can. J. Chem. 1961, 39, 2240-2243.

6. Fox, M.F.; Whittingham, K. P. Component interactions in aqueous dimethyl sulphoxide. J. Chem. Soc., Faraday Trans. 11975, 71, 1407-1412.

7. Clever, H.L.; Pigott, S.P. Enthalpies of mixing of dimethylsulfoxide with water and with several ketones at 298.15 K. J. Chem. Thermodyn.1971, 3, 221-225.

8. Packer, K.J.; Tomlinson, D.J. Nuclear spin relaxation and self-diffusion in the binary system, dimethylsulphoxide (DMSO) + water. Trans. Faraday Soc.1971, 67, 1302 - 1314.

9. Gordalla, B.C.; Zeidler, M.D. Molecular dynamics in the system water- dimethylsulphoxide: A N.M.R. relaxation study. Mol. Phys. 1986, 59, 817- 828.

10. Baker, E.S.; Jonas, J. Transport and relaxation properties of dimethylsulfoxide-water mixtures at high pressure. J. Phys. Chem. 1985, 89 (9), 1730- 1735.

11. Kaatze,U.; Pottel,R.; Schäfer, M. Dielectric spectrum of dimethylsulfoxide/water mixtures as a function of composition. J. Phys. Chem. 1989, 93 (14), 5623-5627.

12. Luzar, A. Dielectric behavior of DMSO-water mixtures: a hydrogenbonding model. J. Mol. Liq. 1990, 46, 221-238.

13. Murthy, S.S.N. Phase behavior of the supercooled aqueous solutions of dimethylSulfoxide, ethylene glycol, and methanol as seen by dielectric spectroscopy. J. Phys. Chem. B 1997, 101 (31), 6043-6049.

14. Murthy, S.S.N. Some insight into the physical basis of the cryoprotective action of dimethyl 
sulfoxide and ethylene glycol. Cryobiology 1998, 36, 84-96.

15. Rasmussen, D.H.; Mackenzie, A.P. Phase diagram for the system water-dimethyl sulphoxide. Nature 1968, 220, 1315-1317.

16. Zhang, Q.; Zhang, X.; Zhao, D.X. Polarizable force field for water-dimethyl sulfoxide systems: I parameterization and gas phase test. J. Mol. Liq.2009, 145, 58-66.

17. Zhang, Q.; Zhang, X.; Zhao, D.X. Polarizable force field for water-dimethyl sulfoxide systems: II properties of mixtures by molecular dynamics simulations. J. Mol. Liq. 2009.145, 67-81.

18. Catalan, J.; Diaz, C.; Garcia-Blanco, F. Characterization of binary solvent mixtures of DMSO with water and other cosolvents. J. Org. Chem. 2001, 66, 5846-5852.

19. Muller, M.G.; Hardy, E.H.; Vogt, P.S.; Bratschi, C.; Kirchner, B.; Huber, H.; Searles, D.J. Calculation of the deuteron quadrupole relaxation rate in a mixture of water and dimethyl sulfoxide. J. Am. Chem. Soc. 2004, 126, 4704-4710.

20. Suresh, S.J. Detailed molecular model for dielectric constant of multicomponent, associating liquids. J. Phys. Chem. B 2004, 108, 715-720.

21. Benjamin, I. Structure, thermodynamics, and dynamics of the liquid/vapor interface of water/dimethylsulfoxide mixtures. J. Chem. Phys. 1999, 110, 8070-8079.

22. De Leeuw, S.W.; Perram, J.M.; Smith, E.R. Computer simulation of the static dielectric constant of systems with permanent electric dipoles. Annu. Rev. Phys. Chem. 1986, 37, 245-270.

23. Ryckaert, J.P.; Ciccotti, G.; Berendsen, H.J.C. Numerical integration of the cartesian equations of motion of a system with constraints: Molecular dynamics of n-alkanes. J. Comput. Phys. 1977, 23. 327-341.

24. Hockney, R.W. The potential calculation and some applications. Meth. Comput. Phys. 1970, 9, 136-210.

25. Cowie, J.M.G.; Toporowski, P.M. Association in the binary liquid system dimethylsulphoxidewater. Can. J. Chem. 1961, 39, 2240-2243.

26. LeBel, R.G.; Goring, D.A.I. Density, viscosity, refractive index, and hygroscopicity of mixtures of water and dimethyl sulfoxide. J. Chem. Eng. Data, 1962, 7, 100-101.

27. Doucet, Y.; Calmes-Perrault, F.; Durand, M.T. Dielectric constants of pure dimethyl sulfoxide and of its aqueous solutions. Compt. Rend. 1965, 260, 1878-1881.

28. Zwanzig, R.W. High-temperature equation of state by a perturbation method. I. nonpolar gases. $J$. Chem. Phys. 1954, 22, 1420-1426.

29. Beveridge, D.L.; DiCapua, F.M. Free Energy via Molecular Simulation: Applications to Chemical and Biomolecular Systems. Ann. Rev. Biophys. Biophys. Chem. 1989, 18, 431-492.

30. Wallqvist, A.; Berne, B.J. Molecular dynamics study of the dependence of water solvation free energy on solute curvature and surface area. J. Phys. Chem. 1995, 99, 2885-2892.

31. Glasstone, S.; Laidle, K.J.; Eyring, H. The theory of rate processes; McGraw-Hill: New York, USA, 1941.

32. Cole, K.S.; Cole, R.H. Dispersion and absorption in dielectrics I. alternating current characteristics. J. Chem. Phys. 1941, 9, 341-351.

33. Davidson, D.W.; Cole, R. H. Dielectric relaxation in glycerol. J. Chem. Phys. 1950, 18, 1417.

34. Debye, P. Polar Molecules; Chemical Catalog. Co.: New York, USA, 1929. 
35. Puranik, S.M.; Kumbharkhane, A.C.; Mehrotra, S.C. Dielectric study of dimethyl sulfoxide-water mixtures using the time-domain technique. J. Chem. Soc. Faraday Trans. 1992, 88, 433-435.

(C) 2009 by the authors; licensee Molecular Diversity Preservation International, Basel, Switzerland. This article is an open-access article distributed under the terms and conditions of the Creative Commons Attribution license (http://creativecommons.org/licenses/by/3.0/). 